\title{
Erratum to: Management of CKD-MBD in non-dialysis patients under regular nephrology care: a prospective multicenter study
}

\author{
Maurizio Gallieni $^{1} \cdot$ Luca De Nicola $^{2} \cdot$ Domenico Santoro $^{3} \cdot$ Gina Meneghel $^{4}$. \\ Marco Formica $^{5}$ - Giuseppe Grandaliano ${ }^{6}$ - Francesco Pizzarelli ${ }^{7}$ - Maria Cossu ${ }^{8}$. \\ Giuseppe Segoloni $^{9}$ - Giuseppe Quintaliani ${ }^{10}$ - Salvatore Di Giulio ${ }^{11}$. \\ Antonio Pisani $^{12}$ - Moreno Malaguti ${ }^{16}$. Cosimo Marseglia ${ }^{13}$ - Lamberto Oldrizzi ${ }^{14}$. \\ Mario Pacilio $^{2}$ - Giuseppe Conte ${ }^{2} \cdot$ Antonio Dal Canton $^{15} \cdot$ Roberto Minutolo $^{2}$
}

Published online: 9 June 2015

(C) Italian Society of Nephrology 2015

\section{Erratum to: J Nephrol \\ DOI 10.1007/s40620-015-0202-4}

In the published article, the name of the author Luca De Nicola is not given correct. The correct name is: Luca De Nicola.

The online version of the original article can be found under doi:10. 1007/s40620-015-0202-4.

Maurizio Gallieni

maurizio.gallieni@fastwebnet.it

1 Nephrology and Dialysis Unit, Ospedale San Carlo Borromeo, University of Milano, via Pio II, 3, 20153 Milan, Italy

2 Second University of Naples, Naples, Italy

3 University of Messina, Messina, Italy

4 Dolo General Hospital, Dolo-Venice, Italy

5 Ospedali di Savigliano e Ceva, ASL Cuneo 1, Cuneo, Italy

6 Department of Medical and Surgical Sciences, University of Foggia, Foggia, Italy
7 S.M. Annunziata Hospital, Florence, Italy

8 SS Annunziata Hospital, Sassari, Italy

9 University of Turin, Turin, Italy

10 S. Maria della Misericordia Hospital, Perugia, Italy

11 S. Camillo Forlanini Hospital, Rome, Italy

12 University Federico II, Naples, Italy

13 C. Poma Hospital, Mantova, Italy

14 Ospedale Fracastoro, San Bonifacio, Italy

15 Fondazione IRCSS Policlinico S. Matteo and University of Pavia, Pavia, Italy

16 Ospedale San Paolo, Civitavecchia, Italy 